\title{
Analysis of Clinical Features and Transmission of Potential of COVID-19 Infection from Pregnant Mother to Baby
}

\author{
Dutta $\mathrm{S}^{*}$ and Bandyopadhyay SK \\ The Bhawanipur Education Society College, India
}

*Corresponding author: Shawni Dutta, The Bhawanipur Education Society College, 5, Elgin Rd, Sreepally, Bhowanipore, Kolkata, West Bengal 700020, Email: shawnidutta83@gmail.com

\section{Mini Review}

Volume 4 Issue 1

Received Date: January 05, 2021

Published Date: January 22, 2021

DOI: $10.23880 /$ jqhe-16000202

\section{Abstract}

Pregnant woman needs special care by an experience doctors. During the time of pregnancy of woman several disorders such as diabetes and high blood pressure may occur and these increase the risk for the baby. So it is needed to consult experienced gynaecologist to get in the best physical condition possible before they become pregnant. It is also needed to assess that if there is any possibility of premature birth. This paper indicates the problems related to pregnant woman during COVID-19 and analysed the chronic illnesses and other health complications.

Keywords: Period of Pregnancy; Fetal Transmission; COVID-19; SARS-CoV-2 and Chronic Illness

\section{Introduction}

Pregnancy is a joyous time in a woman's life. It highlights the woman's amazing creative and nurturing powers. A pregnant woman is a responsible woman and can give best support the health of her future child. This time fetus of woman is growing and it entirely depends on mother's healthy body. It is required that the pregnant women remain as healthy as possible and to be well nourished during the period. The pregnant women should follow proper lifestyle related to health during this period.

World Health Organization (WHO) pointed out on $30^{\text {th }}$ January 2020 that COVID-19 creates respiratory disease caused by the new coronavirus SARS-CoV-2 [1]. Till date it affects the entire world and shows high rate of mortality of human. It is not known that COVID-19 infection may create trouble for pregnant women $[2,3]$. The previous two coronavirus-caused infections, SARS and MERS, have been less effective than COVID-19. Pregnancy is a Severe physiologically stressful conditions arise on the pregnant women. So it renders more susceptible for complications of COVID-19, as compared to a non-pregnant woman.
The awareness of healthcare of pregnant women during COVID-19 is important and their new born babies may be more likely to need specialist care. It is true for pregnant women with COVID-19 alongside other co-morbidities. It is also crucial to know whether or not the pregnant woman has COVID-19. It is also important to recognise the increased stress and anxiety of pregnant women caused by COVID-19. It is required that their partners, children, families and healthcare providers have a role in responding to pregnant women in an appropriate and compassionate way. Pregnant women with COVID-19 with pre-existing medical conditions, such as diabetes or chronic high blood pressure are also more likely to suffer severe health complications due to COVID-19. So it is important to study the effect of COVID-19 on Pregnant Women. This paper made an initial study on this situation and it is true that a thorough study is required for the effect of COVID-19 on pregnant women.

The panic and worry over this new virus have no cure. Pregnant women are a special group for consideration under the current situation because the levels of the hormones estrogen and progesterone go up during pregnancy. It causes the upper respiratory tract to get more easily infected. 
Pregnant women makes them more hypoimmunity due to the immunological tolerance status increases. The pivotal events in a woman's life are Pregnancy, birth and parenting. These are bio-psychosocial events. There is no turning back to a prepregnant psychology once a woman has been pregnant. The desire for motherhood appears to be only essential for female during pregnancy. The pregnancy is viewed as a time of crisis and a critical phase for woman. Pregnancy is also an opportunity for growth and reworking of self-concept for many women.

The contribution of this paper may be summarized as follows

- Consider the pregnant women as the target group for analysis.

- Analyse their mental health status for understanding the well-being of the mother.

- Analyse physical strength of the considered group with respect to Hemostasis disease.

For perceiving the mental health status and physical strength of target group, existing research works are illustrated.

\section{Related Works and Study of Behaviour of Pregnant Women}

A brief summary of existing studies are given in this section. For simplicity and better understanding, the objective of this current study is divided into two subsequent portions. The first subsection gives a prominent idea regarding the impact of Covid-19 and mental health status grown in pregnancy period. The second subsection illustrates the negative effect of the disease known as Hemostasis during the pregnancy period.

\section{Mental Health of Pregnant Women During Covid-19}

WHO guides on the prenatal care has reinforced the importance of knowing and intervening on psychosocial variables in pregnant women and new born baby. It is the the aim of optimizing the physical and psychosocial well-being of the mother and the baby. So it requires special attention for pregnant woman in relation to prevention, diagnosis and management [3]. 10\% of pregnant women suffer from mental disorder globally in normal times. The reason is primarily for depression and it is even higher if there is any complication [4].

Covid-19 pandemic may increase mental anxiety of pregnant women. The restricted access to mental health services may further enhanced the anxiety. It is a major public health challenge. It requires appropriate and timely health care support to avert adverse health outcomes. Almost $35 \%$ of the pregnant mothers were self-isolating to prevent themselves from getting COVID-19 transmission. This result is obtained after proper survey over pregnant woman [5].

A survey is collected the details of 102 pregnant women, and a control group of 102 non-pregnant women [6]. During the quarantine period all women showed a gradual increase in psychopathological indicators and a decrease in positive affect. A more pronounced increase in depression, anxiety and negative affect of pregnant women than the non-pregnant women is visualized in this period. Pregnant women always afraid of negative thinking and in turn it increases anxiety.

A study was made over 100 pregnant women [7]. It is based on in the first, second, and third trimester of pregnancies, respectively. There is no history of any type of depression prior pregnancy or any psychological disorders. A moderate psychological impact on pregnant women with a mean IES-R score of $36.9+10.1$ during COVID-19. Out of which $53 \%$ of the enrolled women have a severe psychological impact. This study foresees that there is a serious need of study over pregnant women during COVID-19 [7].

\section{Hemostasis and Pregnancy}

Pregnancy is characterized by prothrombotic condition and it creates procoagulant imbalance among pregnant woman which serves to protect women from bleeding but increases their risk for venous thromboembolism (VTE). Coronavirus disease 2019 (COVID-19) does not spared pregnant women with regards to VTE $[8,9]$. Hemostasis is the physiological process. Normal blood flow elsewhere in the circulation is maintained other than injury portion where bleeding is stopped [10]. The hemostatic status of pregnant women with COVID-19 has been studied [11]. This study was similar in pregnant women with COVID-19 who were asymptomatic or had mild symptoms and in pregnant women without infection [11].

Initially it is thought that pregnant women with COVID-19 were no more likely to develop severe morbidity or die. The recent reports suggest that a subset may develop multiorgan failure and even die. It is found that healthy pregnant women have evidence of increased generation of thrombin and a prothrombotic state as well as increased intravascular inflammation. It is exaggerated in the context of infection, such patients may be at an increased risk for thrombosis when affected by COVID-19 [12].

For management of COVID-19 coagulopathy the International Society of Thrombosis and Haemostasis has generated algorithm for finding relation in between COVID-19 and change of state of pregnant women. It is recommended that low-molecular-weight heparin be considered in all such patients. It is also needed that a coagulation profile to be detected the presence of subclinical disseminated 
intravascular coagulation and the use of low-molecularweight heparin for the prevention of thromboembolic disorders should be considered and discussed with physicians and patients [13].

In order to avoid transmission of the concerned viral infection, precaution needs to be assured. Infections due to contact via infectious vaginal secretions during birth, or via respiratory secretions after birth should be monitored to avoid transmissions. Thus, a mother who has confirmed COVID19 or is a person under investigation should be isolated from her baby until the mother is free from any possible transmission danger [14].

\section{Conclusion}

This study focuses on considering the mental health status as well as physiological effects of pregnancy. An impact of COVID-19 pandemic situation on the pregnant women is perceived in this paper. Hemostasis is a serious condition that may be developed during pregnancy which in turn may create a life-threatening stage to the child as well as mother. This paper has given its justification on the observed impacts of the considered events. Existing studies are utilized and reviewed in order to justify the influence of current pandemic situation upon the new-life givers. Analysis of this perspective is necessary because it defines the wellbeing of motherhood. Early detection of these considered perspectives during this crucial period is clinically important. However, this paper can even be extended by considering other relevant and influential factors that may have effect during pregnancy.

\section{References}

1. Nishiura, Hiroshi (2020) The extent of transmission of novel coronavirus in Wuhan, China, 2020. J Clin Med 9(2): 330 .

2. Lee, Dominic TS (2006) Psychological responses of pregnant women to an infectious outbreak: a casecontrol study of the 2003 SARS outbreak in Hong Kong. Journal of psychosomatic research 61(5): 707-713.

3. World Medical Organization (2020) WHO recommendations on antenatal care for a positive pregnancy experience.

4. Liang Nan Z (2020) Mental health care for pregnant women in the COVID-19 outbreak is urgently needed." Women and birth: journal of the Australian College of Midwives S1871-5192(20): 30210-30209.

5. Corbett, Gillian A (2020) Health anxiety and behavioural changes of pregnant women during the COVID-19 pandemic. European Journal of Obstetrics, Gynecology, and Reproductive Biology 249: 96-97.

6. Morales L, Hernán (2020) Mental health of pregnant women during the COVID-19 pandemic: a longitudinal study. Psychiatry research 295: 113567.

7. Saccone, Gabriele (2020) Psychological impact of coronavirus disease 2019 in pregnant women. American Journal of Obstetrics \& Gynecology 223(2): 293-295.

8. James AH, Jamison MG, Brancazio LR, Myers ER (2006) Venous thromboembolism during pregnancy and the postpartum period: incidence, risk factors, and mortality. Am J Obstet Gynecol 194(5): 1311-1315.

9. Martinelli I, Ferrazzi E, Ciavarella A (2020) Pulmonary embolism in a young pregnant woman with COVID-19. Thromb Res 191: 36-37.

10. Andrew JG (2011) Continuing education course \#2: current understanding of hemostasis. Toxicologic pathology 39(1): 273-280.

11. Ciavarella, Alessandro (2020) Hemostasis in pregnant women with COVID-19. International Journal of Gynecology \& Obstetrics.

12. Renzo D, Carlo G, Giardina I (2020) Coronavirus disease 2019 in pregnancy: consider thromboembolic disorders and thromboprophylaxis. American Journal of Obstetrics \& Gynecology 223(1): 135.

13. Jecko $\mathrm{T}$ (2020) ISTH interim guidance on recognition and management of coagulopathy in COVID-19. Journal of Thrombosis and Haemostasis 18(5): 1023-1026.

14. Guillaume F (2020) Guidelines for pregnant women with suspected SARS-CoV-2 infection. The Lancet Infectious Diseases 20(6): 652-653. 